\author{
Voix plurielles \\ Volume 3, Numéro 2 : septembre 2006
}

Sylvain Rheault

\title{
Témoignage indicible de la violence : le cas du poilu de la Grande Guerre
}

Citation MLA : Sylvain Rheault. «Témoignage indicible de la violence : le cas du poilu de la Grande Guerre .» Voix plurielles 3.2 (septembre 2006). 


\title{
Témoignage indicible de la violence : le cas du poilu de la Grande Guerre
}

\author{
Sylvain Rheault \\ Université de Regina
}

Septembre 2006

\begin{abstract}
Résumé analytique
$\mathrm{L}$

e témoignage indicible tente de rapporter des événements pour lesquels les lecteurs potentiels n'ont aucun point de référence. Une analyse des stratégies d'écriture à partir des pôles de l'énonciation permet de mettre en lumière un dilemme terrible : raconter, décrire au moyen des formes qui existent, engage malgré lui le témoin à distiller des événements une certaine beauté. Ce qui était indicible risque de devenir épique. Pour communiquer la vérité d'une violence comme n'en a jamais vue le public, le témoignage indicible est condamné à ne pas s'engager dans la voie de l'esthétique.
\end{abstract}

Le témoignage indicible se définit comme la démarche courageuse d'un individu qui cherche à imprégner la conscience collective d'événements qui dépassent les conceptions les plus perverses de l'imagination et qui, pour cet individu, sont lourdement lestés d'expériences traumatisantes. Cathy Caruth, dans Unclaimed Experience, dégage dans la blessure une histoire en attente d'être révélée :

it [trauma] is always the story of a wound that cries out, that addresses us in the attempt to tell us of a reality or truth that is not otherwise available. (4)

Les humiliations dégradantes de la guerre se traduisent souvent par un mutisme impénétrable, sinon par la folie, dont les victimes de psychose traumatique (shell shock) ne sont qu'un exemple parmi d'autres.

Drieu La Rochelle, dans La Comédie de Charleroi, traduit l'angoisse du témoin face à son indescriptible expérience de la guerre des tranchées. Interpelant les humains, il s'interroge :

Comprennent-ils ? Savent-ils ? Se rappellent-ils ? Réfléchissent-ils ? Ces femmes ont-elles jamais deviné ? Ces hommes, s'ils y furent, peuvent-ils raconter? (150)

Le premier conflit mondial s'est révélé d'une ampleur sans précédent tant par le nombre d'hommes appelés sous les drapeaux que par les ressources consommées et le niveau technologique atteint. Le poilu de la Grande Guerre a subi des épreuves indescriptibles : sa chair a été meurtrie, 
son corps a été broyé, son âme a été souillée et les rapports qu'il entretenait avec les autres humains ont été sapés. Il y avait trop de souffrances. Il n'y avait pas moyen de décrire l'expérience.

Dans le cadre du présent article, sera qualifié d'indicible le témoignage portant sur un événement dont la réalisation et les résultats dépassent en portée les pires horreurs qu'il est possible à un public d'imaginer et qui, pour l'individu qui le subit, représente une humiliation profonde. Narrer la peur ressentie en affrontant un nid de mitrailleuses ou la décapitation d'un ami cher par un bombardement de tranchées, voilà des exemples de témoignages indicibles. Jean Norton Cru, dans Témoins, résume assez bien les difficultés qu'éprouve le soldat de la Grande Guerre à relater l'expérience vécue :

[...] l'impuissance du vocabulaire à désigner les choses autrement que comme des abstractions, [...] l'impuissance du soldat à traduire en mots ce qui est à peu près intraduisible parce que les mots sont destinés à être compris tandis que les choses du front, serties dans leur atmosphère, ne peuvent être perçues que par les cinq sens et surtout, et par contrecoup, par l'âme. (225-230)

Jean Norton Cru, lui-même un ancien combattant, s'était attelé à coter selon leur valeur documentaire tous les récits portant sur la Grande Guerre écrits jusqu'en 1928. C'est à la mesure de son propre calvaire dans les tranchées que Norton Cru a pu «méthodiquement » estimer le degré de "vérité » des textes. Si on peut questionner l'objectivité de sa démarche critique, son ouvrage, Témoins, n'en reste pas moins une contribution importante à la recherche sur les textes de la Grande Guerre. Entre autres, les observations et les insistances de Jean Norton Cru ont permis de mettre à jour certains « pôles énonciatifs » dont nous nous servirons.

Le présent article propose un classement en catégories et en pôles les choix énonciatifs des témoins. Nous adopterons les catégories de l'énonciation tirées de la grille de classement de La Clé - Répertoire de procédés littéraires de Dupriez et Rheault (1998). Inspirés des six pôles de l'énonciation de Jakobson, il s'agit de la situation, de la visée argumentative, du contact (ou ton), du locuteur, du destinataire et du façonnement esthétique. Pour chaque catégorie, deux pôles seront proposés. La lecture proposée ne fait pas dans la nuance. Il s'agit de repérer les positions les plus extrêmes pour chacun des aspects de l'énonciation et d'observer auquel de ces pôles tendent préférentiellement les témoignages du corpus. Pour les besoins d'un article court, ne seront retenus qu'une poignée d'auteurs connus ayant participé à des combats : Barbusse, Céline, Cocteau, Cendrars, Dorgelès, Drieu la Rochelle, Giono ainsi que quelques lettres de poilus.

\section{La situation du témoignage indicible}

La situation englobe le signifié conceptuel et donne un contexte réel à la communication. Le texte peut être caractérisé par sa relation avec l'environnement lors de la rédaction du témoignage. On peut distinguer ici deux pôles : la proximité à l'événement ou la distance à celui-ci.

On pourrait nuancer en spécifiant la proximité ou l'éloignement au moyen des dimensions physiques et temporelles de la situation. On aurait dans un premier cas, une proximité du témoignage à l'événement qui serait à la fois physique et temporelle. Le témoin transcrit son expérience 
alors qu'il la vit. On aurait ensuite le cas d'un témoignage offrant une proximité temporelle avec l'événement, mais assorti d'une distance physique, dans l'éventualité où le témoin transcrirait ses souvenirs peu de temps après l'événement, mais dans un lieu éloigné. Vient ensuite le cas du témoignage où la proximité physique doit composer avec une distance temporelle, à l'occasion d'un retour sur les lieux de l'événement, longtemps après, alors que le témoin cherche à se remémorer les faits. Enfin, la transcription des faits dans le cas d'une distance à la fois physique et temporelle permet au témoin de recourir à sa maturité et à ses expériences postérieures pour réexaminer les événements.

Plus que les aspects de temporalité et de lieux, ce sont les positions de proximité et de distance qui présentent le plus d'intérêt pour l'analyse. Lors d'une conférence portant sur la question : « Qu'est-ce qu'un bon témoin du front ? », Jean Norton Cru a énoncé cette première condition :

I - Il faut que le récit soit fondé sur un journal tenu au jour le jour, avec notation immédiate des faits et fixation spontanée des émotions toutes fraîches. À défaut de journal, les notes intermittentes doivent être datées. ${ }^{1}$

Selon ce critère, le témoignage tiendrait sa validité en s'établissant sur un document qui aurait été écrit dans les plus brefs délais après l'événement et qui devrait comporter un minimum de notations chronologiques.

Rousseau, dans Le Procès des témoins de la Grande Guerre. L'Affaire Norton Cru, critique cette « condition de la véracité du témoin du front »(229) en rappelant que la proximité à l'événement impose des contraintes périlleuses. Lorsqu'il endure un bombardement d'artillerie ou lorsqu'il attaque, le poilu préfère assurer sa survie plutôt que de mettre par écrit ce qu'il voit ou ressent. Même lors des périodes d'accalmie, les conditions de vie dans les tranchées, sujettes aux privations matérielles et aux rigueurs météorologiques, ne permettaient guère de tenir un carnet ou de produire mieux qu'une correspondance succincte. La spontanéité affleure dans les exemples qu'on peut trouver de ce genre d'écrit :

Voilà le réveil. Il gèle dehors. Au sortir des rêves de la nuit, devant ce joli temps de gel, et à me retrouver ici je sens revenir le cafard. ${ }^{2}$

Ce ne sont là, explique Rousseau, que des soucis immédiats (202). Il faut aussi mentionner que la correspondance faisait l'objet d'une censure tout autant pour garantir le secret sur la disposition des troupes que pour maintenir le moral de la population. Si la proximité à l'événement favorise la validité du témoignage, en revanche elle en limite aussi le champ, donc les possibilités de saisir les faits dans toute leur complexité. Le potentiel historique du témoignage se trouve ainsi sérieusement handicapé.

Lorsque le témoignage apparaît après un certain temps, il est possible à l'auteur d'étoffer sa narration des événements. Il en résulte des textes ayant plus de maturité, comme des mémoires ou des romans. Si certains témoignages ont été publiés pendant la guerre ou tout de suite après, comme les oeuvres de Barbusse en 1916 et de Dorgelès en 1919, d'autres l'ont été de nombreuses années plus tard, comme Giono en 1931, Céline en 1932, Drieu en 1934, Cendrars en 1946 et Aragon en 1956. Les récits « proches » s'apparentent davantage à des journaux tandis que les plus 
« distants » se présentent comme des fictions. De plus, le lecteur, et l'historien aussi sans doute, sera sensible à la date de publication de « 1916 » qu'on trouve en ouvrant le livre de Barbusse. On a l'impression d'aborder l'histoire, tandis qu'on lira les poèmes d'Aragon sur le même sujet, écrits en 1956, d'abord pour leurs qualités littéraires.

Entre les pôles de la proximité et de la distance à l'événement, le témoignage indicible semble osciller plus volontiers vers la proximité, afin de mieux faire sentir au lecteur " qu'on y était». C'est aussi que la stratégie de la distance favorise ce qu'on pourrait appeler le « témoignage épique ». Ce concept, qui sera élaboré au fil de l'article, se présente en opposition au témoignage indicible et se caractérise par la primauté de l'esthétique sur la vérité.

\section{La visée argumentative du témoignage}

La visée argumentative définit le véritable enjeu de la communication. Distincte de l'énoncé, la visée permet de conceptualiser des procédés comme ceux qui s'apparentent au mensonge. Les pôles à distinguer ici sont la visée égoïste ( « essentiellement pour son propre bénéfice ») et la visée altruiste (« essentiellement pour le bénéfice des autres »).

Le soldat peut se taire devant l'inavouable humiliation qu'il a subi au front, et cela n'équivaut pas à nier l'événement. À propos de ce mutisme, Norton Cru condamnait ceux des poilus qui ne témoignaient pas (36) et les accusait de contribuer ainsi à maintenir le public dans l'ignorance des méfaits et des horreurs de la guerre. D'autre part, comme en temps de guerre les projecteurs de l'histoire sont braqués sur les événements militaires, le poilu peut chercher, par son témoignage, à se greffer à l'histoire, comme on grave son nom sur un monument célèbre. Rousseau avance que « Norton Cru invente un droit nouveau : le droit à l'histoire pour la piétaille » (64). Entre le divertissement et l'information, un témoignage qui relève du premier manifeste une visée égoïste en ce sens que le témoignage pourrait chercher à rendre populaire son auteur. Cela explique pourquoi certains historiens jugent suspects tous les témoignages présentant des qualités littéraires (237). Lorsqu'un auteur se mêle d'interpréter les événements on le soupçonnera de chercher à promouvoir une idéologie, comme le pacifisme ou le communisme, de manière soit à changer le système de valeur du destinataire, soit à justifier les valeurs collectives en usage.

Dans une autre lettre, Barbusse voit dans les socialistes, « mathématiquement, fatalement, le seul recours possible contre les guerres futures. Tout le reste est chimère $\rangle^{3}$

C'est en souhaitant voir l'émergence du communisme dans la société française que Barbusse rédige Le Feu :

Ah ! vous avez raison, pauvres ouvriers innombrables des batailles, vous qui aurez fait toute la guerre avec vos mains, toute-puissance qui ne sert pas encore à faire le bien, [...] (283)

L'idéologie socialiste qui alimente Barbusse laisse ici des traces dans l'écriture. Dire « ouvriers » plutôt que « soldats » n'est pas innocent et semble viser une classe sociale bien précise. Le rapprochement de « guerre » et « mains » renforce cette image de conflit de classes en évoquant les travailleurs manuels mobilisés. Enfin, la potentialité contenue dans « qui ne sert pas encore à faire le bien » suggère une révolution qu'il reste à faire. 
Le pôle de la visée argumentative altruiste, consiste pour un témoin à prendre la peine d'attester de l'événement afin de le porter à la connaissance de la conscience collective. Rousseau rappelle que « lutter contre la guerre consiste tout d'abord à lutter contre l'ignorance » (93). Le témoin doit s'effacer devant l'événement et en donner au lecteur la plus juste représentation possible. Norton Cru dénonce avec autant de vigueur les faits mal rapportés (ou inventés, ce qui est pire) que les faits exagérés, puisque l'un et l'autre, selon lui, seront discrédités aux yeux des historiens.

Le témoignage indicible a intérêt, on s'en rend compte, à adopter les caractéristiques du pôle des visées altruistes. Le témoignage épique, au contraire, veut intéresser avant que d'informer.

\section{Le statut de l'auteur du témoignage}

Dans le contexte de la Grande Guerre, on peut se demander, à propos de l'auteur du témoignage, s'il a été un combattant ou non. On aura ainsi les pôles de l'auteur non-combattant et de l'auteur combattant.

Le lecteur accordera peu de crédibilité historique aux témoignages des non-combattants. Par exemple, on sait en parcourant sa biographie que Jean Cocteau a été brièvement ambulancier pendant la Grande Guerre. Étant donné qu'il n'a pas eu de contact direct avec le front, les critiques apprécient son Thomas l'imposteur surtout comme une fiction littéraire, un « jeu » dirait Rieuneau (104) et ne s'y réfèrent pas en tant que témoignage. On juge que le texte offre une habile reconstitution historique, sans plus, comme le font aussi des oeuvres telles Salammbô de Flaubert ou La Bataille de Patrick Rambaud. De même, l'historien, en tant qu'auteur non-combattant, se voit contraint de quêter auprès des vrais témoins la validité de ses reconstitutions.

Quant au combattant de la Grande Guerre, qui est-il au juste ? Jean Norton Cru dresse cette fiche d'identification:

tout homme qui fait partie des troupes combattantes ou qui vit avec elle sous le feu, aux tranchées et au cantonnement, à l'ambulance du front, aux petits états-majors. [...] La guerre elle-même a imposée cette définition fondée sur l'exposition au danger et non plus sur le port des armes qui ne signifie plus rien. (10)

À noter que la distinction vainqueur/vaincu se voit éludée de la définition. Aragon avait remarqué cette omission dans les récits de guerre :

Il n'y a pas d'exemple d'une victoire qui ait inspiré un roman où on voie la guerre, la guerre victorieuse. [...] L'autre guerre, celle qu'on appelait la Grande, a produit un tas de romans, pendant et après : mais du Feu à Verdun, aucun ne décrit la victoire, tous sont centrés sur l'horreur de la vie des tranchées. ${ }^{4}$

Il en résulte un mouvement de sympathie envers celui qu'on affuble du nom « d'ennemi ». En effet, cet ennemi ne souffre-t-il pas pareillement de «l'exposition au danger ? » Le soldat de ce côté-ci du no man's land en vient à projeter ses propres sentiments sur le soldat de la tranchée d'en face : 
Et puis mon pauvre Maurice, il faut réfléchir que les Prussiens sont comme nous. Vois-tu qu'un garçon prussien écrive à son père la même chose que toi et qu'il lui demande un képi de Français, et si ce papa prussien rapportait un képi de Français à son petit garçon et que ce képi fut celui de ton papa ${ }^{5}$

Au lieu de raconter des succès collectif, le témoin transmet une expérience individuelle. Avec la stagnation du front, les charges héroïques ne sont plus à envisager, l'essentiel est de survivre. Le soldat se découvre la dupe des illusions de conquête qu' on lui faisait miroiter et se rend compte qu'il n'est, après tout, qu'une victime. Dans les conditions uniques de la Grande Guerre, la souffrance éprouvée n'est plus perçue comme un sacrifice pour la collectivité mais comme une humiliation individuelle.

Pour en revenir à la fiche d'identification de Norton Cru, il faut noter que le témoignage doit venir du « bas » (des poilus) et non pas du « haut » (des généraux) de la hiérarchie militaire, c'està-dire des individus exposés aux effets destructeurs des armes. Norton Cru ajoute que : " toute histoire militaire vue de haut, conçue en partie d'échecs, faite d'après les documents d'état-major [...] est une agréable illusion. » (15)

La démocratie et l'éducation semblent avoir été des catalyseurs aux témoignages des poilus puisque, pour la première fois en France, beaucoup d'appelés sous les drapeaux avaient une formation scolaire suffisante pour communiquer leurs impressions sous forme de récit. Ce phénomène, nouveau en France en 1914, avait déjà été observé lors de la guerre de Sécession, comme le remarque Paul Fussell dans The Great War and Modern Memory :

The American Civil War was the first, Theodor Ropp observes, « in which really large numbers of literate men fought as common soldiers ». (157)

En France, les réformes de Jules Ferry rendant l'enseignement obligatoire avaient été votées dans les années 1880, soit quelques années après la guerre franco-prussienne de 1870-1871. La mobilisation d'une masse de jeunes hommes capables de lire et d'écrire allait littéralement produire une armée de témoins lors de la Grande Guerre. Certaines études suggèrent qu'environ 1000 écrivains auraient combattu pour la France. ${ }^{6}$

Après ce qu'on vient de voir concernant l'auteur, on s'attendrait à ce que plus ce dernier a pu être exposé au feu, plus son témoignage aura de valeur. Cependant, malgré l'insistance de Jean Norton Cru sur ce point, ce sont surtout les oeuvres ayant des qualités littéraires qu'on réédite. On compte à la Bibliothèque nationale de France 29 éditions du Feu de Barbusse, dont la plus récente date de 1996, et 21 éditions des Croix de Bois de Dorgelès, dont la plus récente date aussi de 1996. Les livres de ces deux auteurs avaient pourtant été classés dans les médiocres par Norton Cru, alors que pour les auteurs jugés bons, et qui sont aussi considérés comme littéraires, comme Pierre Chaine, on ne compte que 10 éditions des Mémoires d'un rat, même si la plus récente a été publiée en 2000. Il faut rappeler que Barbusse militait activement pour le communisme et que Dorgelès siégeait à l'académie Goncourt. Le talent et le prestige social de l'auteur semblent avoir été des facteurs plus déterminants pour la postérité des oeuvres que l'authenticité du témoignage. 


\section{Le ton du témoignage}

Le ton du témoignage détermine la qualité du contact qui s'établit entre l'auteur et son public. Les deux pôles contraires à considérer ici sont, d'une part, le ton qui envisage le public comme inférieur, et, d'autre part, le ton qui envisage le public comme supérieur (ou égal) au témoin.

Dans le cas où le public est considéré comme inférieur, le locuteur adopte un ton paternaliste. Xavier Roux, dans L'Âme de nos soldats d'après leurs actes et leurs lettres, un ouvrage publié en 1914, expose ses intentions au début du livre :

J'ai cueilli de ma main vieillissante et j'ai réuni en gerbes les pensées, les sentiments, les espérances et tous les gestes qui sont des vertus, des soldats, vos fils ou vos époux. (7)

Xavier Roux dédie ainsi son livre aux femmes dont les fils ou les maris sont aux armées. Il se pare du prestige de l'âge et il assume que les lectrices ne savent absolument rien du front. Cependant, Roux lui-même ne sait des combats que ce qu'il en a lu dans les journaux et dans les lettres qu'il a eu entre les mains. S'il avait été vétéran de la guerre de Crimée ou de la guerre franco-prussienne, il n'aurait pas manqué de le faire remarquer. Quoi qu'il en soit, l'effet produit est que les événements apparaissent grossièrement déformés par le filtre de l'ascendant que se donne l'auteur. Il use d'euphémismes pour ne pas effaroucher son public : « 'trouer' des Prussiens » (110). À force de ménagement et d'adoucissement, le témoignage qu'il promettait s'en trouve dilué. Bref, l'adoption d'un ton supérieur, qui a pour effet de donner un caractère épique au texte, disqualifie la validité du témoignage.

En parcourant le corpus, on constate que plusieurs récits s'ouvrent dans les tranchées, comme le Feu de Barbusse ou les Croix de bois de Dorgelès. Les poilus s'expriment en argot et aucun effet des armes sur les corps n'est ménagé au lecteur. Le ton adopté invite le public à devenir un membre à part entière de l'escouade. Giono, en faisant commencer Le Grand Troupeau dans un village qu'on a dépouillé de ses hommes, invite ainsi les civils à s'identifier à l'histoire. Le public se voit considéré comme l'égal des personnages ou du narrateur. La stratégie du ton égalitaire permet d'établir la confiance nécessaire pour la présentation du témoignage indicible.

\section{Le destinataire du témoignage}

Les deux pôles à distinguer ici sont l'individu d'une part (destinataire singulier) et le grand public d'autre part (destinataire universel). Il y a cependant une position intermédiaire à considérer, soit le public d'anciens combattants (destinataire militaire), qui forme une sous-collectivité importante pendant et après la Grande Guerre. Il peut s'agir de frères d'armes qui ont combattu dans la même guerre, ou de combattants qui ont eu l'expérience du feu dans une autre guerre ou même de civils qui, sans avoir participé directement aux combats, n'en ont pas moins subi les contrecoups pour avoir été au mauvais endroit au mauvais moment.

Le témoignage qui n'envisage comme destinataire que le témoin lui-même se présente comme une production qui rapporte des étapes de service et des soucis intimes. Il s'agit d'un simple aidemémoire qui n'a souvent de valeur ni pour la littérature ni pour l'historien. Rousseau statue que « les journaux de marche n'offrent d'intérêt que pour le signataire seulement » (202). 
La tentation est grande pour le témoin de s'adresser au destinataire qui a vécu les mêmes expériences, et qui peut aisément percevoir ce qui est indicible derrière quelques mots jetés sur une page :

Mais offrez ces livres [qui offrent de nombreux détails du front] à une femme, à un jeune homme, ils risqueront fort d'être rebutés par l'abus des petits détails, souvent incompréhensibles pour eux, par la monotonie apparente de l'analyse ou du récit. Ce sont là des livres pour anciens combattants. ${ }^{7}$

Rieuneau note que le public militaire a assuré le succès des romans de guerre ${ }^{8}$ Bien sûr, il y aura de « nouveaux » anciens combattants après chaque nouvelle guerre, mais ce groupe de lecteur ne représentera jamais qu'une mince proportion du lectorat potentiel, proportion qui s'amenuisera à mesure que vieilliront les vétérans. On pourrait soutenir que le destinataire militaire ne constitue en fait qu'une simple extension du destinataire singulier. Il faut aussi se demander ce que vaut un témoignage s'il ne peut être compris que par un nombre limité d'initiés. D'ailleurs, il existait une incompréhension profonde entre les civils et les militaires :

Nous [les soldats] échangions nos impressions à haute voix, et les gens ne comprenaient pas, pas plus notre langage que notre émotion. ${ }^{9}$

Que veux-tu, j'ai constaté, comme tous mes camarades du reste, que ces deux ans de guerre avaient amené petit à petit, chez la population civile, l'égoïsme et l'indifférence et que nous autres combattants nous étions presque oubliés. ${ }^{10}$

Cela laisse présager des obstacles qu'il y pour un poilu à communiquer son expérience à un non-combattant. Le baptème du feu crée une distinction sociale si nette qu'on en trouve aujourd'hui encore des traces dans la collectivité : en France, on réserve des sièges de métro et d'autobus aux anciens combattants, au Canada, un ministère leur est consacré, qui s'occupe de leurs besoins spécifiques, etc.

Comment aborder un public qui n'a pas combattu dans une guerre et qui, dans dans certains cas, n'en a pas vécu non plus, c'est-à-dire qui n'a absolument aucun point de repère pour comprendre l'événement. Le public de l'avenir, à qui il ne restera que des documents écrits lorsque tous les témoins vivants de la Grande Guerre auront disparu, exemplifie ce cas. Ici apparaît un sérieux dilemme. Si, en vue de joindre le plus large public possible, le témoignage doit se limiter aux références et aux capacités d'entendement du public moyen, comment peut-il réaliser son objectif de montrer le vrai visage de l'événement si ce n'est en contraignant le destinataire à évoluer en territoire inconnu, au risque de le rebuter.

Ainsi, le témoignage indicible n'a d'autre choix que de présenter au destinataire un contenu qui risque de dépasser ses références et ses capacités d'entendement. Le témoignage épique, quant à lui, assure sa popularité en présentant au destinataire un contenu qui correspond à ses références et à ses capacités d'entendement.

\section{Façonnement esthétique du témoignage}

Le façonnement esthétique représente les choix effectués en regard du travail de l'artiste. On 
l'a vu, pour certains critiques comme Norton Cru, la forme n'importe pas tant que le degré de conformité du témoignage à la réalité. En fait, ces deux pôles du « vrai » et du « beau » hantent tous les aspects de l'énonciation. On a vu plus tôt comment la spontanéité de la proximité s'opposait à la maturité de la distance, combien il est difficile de concilier auprès du public l'utilité et la popularité, et bien d'autres alternatives où les choix peuvent s'avérer aussi douloureux que dramatiques. Julien Benda, dans une reprise syntaxique habile, contraste le « rien n'est beau que le vrai » d'avant au « rien n'est vrai que le beau » d'aujourd'hui. ${ }^{11}$ Si le témoignage apparaît « indicible », c'est entre autres parce que sa vérité n'engendre pas sa propre beauté, et qu'il n'y a pas de contenant tout prêt à recueillir la vérité toute nue. L'exercice le plus difficile du témoignage consiste à inventer sa propre forme. Barbusse a été le premier à publier, et donc a pu servir de modèle aux récits de guerre à venir. Il lui avait fallu inventer un genre sur mesure, ce que l'on constate à la lecture du sous-titre du Feu : Journal d'une escouade. Un journal rapporte des événements « vrais » du quotidien, de la routine et on s'attend à suivre les faits d'une dizaine de personnages puisqu'il s'agit d'une escouade.

Le témoignage indicible oblige à inventer péniblement de nouvelles formes tandis que le témoignage épique, au contraire, se contente d'exploiter les genres éprouvés.

\section{Conclusion}

Du point de vue des matériaux de l'énonciation, les pôles convenant au témoignage indicible s'écartent radicalement de ceux qui exprimeraient le témoignage épique. Une synthèse en deux phrases s'énoncerait comme suit : le témoignage épique préfère la distance, qu'elle soit physique ou temporelle, se construit autour de visées égoïstes, encourage le locuteur à l'invention, adopte un ton supérieur, s'ajuste aux attentes du public et profite de formes déjà populaires. Le témoignage indicible préfère la proximité, est sous-tendu de visées altruistes, astreint le locuteur à la vérité, adopte un ton égalitaire, astreint le public à tenter de saisir ce qui le dépasse et doit péniblement inventer sa propre forme.

Les stratégies énonciatives relevées dans les textes suggèrent que le témoignage épique s'ajuste parfaitement aux attentes esthétiques de la conscience collective. Les structures énonciatives usuelles semblent favoriser la création et la diffusion du beau au détriment du vrai. Cela n'est pas sans rappeler le personnage d'Ulysse, dans Naissance de l'Odyssée de Giono, qui découvre que son art de rendre un récit intéressant lui attire bien plus de sympathies que s'il avait vraiment réalisé les exploits qu'il raconte.

Au contraire, le témoignage indicible apparaît comme une entreprise semée d'embûches. On décèle de la résistance dans les structures énonciatives : le témoignage doit s'établir à rebroussepoil de chacun des matériaux de l'énonciation afin de les harnacher à une vérité qui ne semble jamais exprimée avec une beauté satisfaisante.

\section{Bibliographie - Corpus}

Guéno, Jean-Pierre, et Yves Laplume (Éds). Parole de Poilus. Paris : Librio, 1998. Aragon, Louis. Le Roman inachevé. Paris : Gallimard, 1956.

Barbusse, Henri. Le Feu. 1916. Paris : Flammarion, 1965. 
Céline, Louis-Ferdinand. Voyage au bout de la nuit. 1932. Paris : Gallimard, 1996.

Cendrars, Blaise. La Main coupée. 1946. Paris : Denoël, 1991.

Cocteau, Jean. Thomas l'imposteur. 1923. Paris : Gallimard, 1943.

Dorgelès, Roland. Les Croix de bois. 1919. Paris : Albin Michel, 1945.

Drieu La Rochelle, Pierre. La Comédie de Charleroi. 1934. Paris : Gallimard, 1970.

Giono, Jean. Naissance de l'Odyssée. 1930. Paris : Gallimard, 1978.

Roux, Xavier. L'Âme de nos soldats d'après leurs actes et leurs lettres, Paris : Société française d'imprimerie et de librairie, 1914.

\section{Bibliographie - Oeuvres critiques}

Caruth, Cathy. Unclaimed Experience. Baltimore : John Hopkins University Press, 1996.

Dupriez, Bernard et Sylvain Rheault. La Clé - Répertoire de procédés littéraires. Montréal : C.A.F.É, 1998. <http://www.cafe.edu/cle>.

Fussell, Paul. The Great War and Modern Memory. Oxford : Oxford University Press, 1977.

Norton Cru, Jean. Témoins. Essai d'analyse et de critique des souvenirs de combattants édités en français de 1915 à 1928. Paris : Les Étincelles, 1929.

Rieuneau, Maurice. Guerre et révolution dans le roman français 1919-1939. Paris : Klincksieck, 1974.

Rousseau, Frédéric. Le Procès des témoins de la Grande Guerre. L’Affaire Norton Cru. Paris : Seuil, 2004.

\section{Notes}

${ }^{1}$ Jean Norton Cru, cité par Rousseau (230).

${ }^{2}$ Étienne Tanty, cité par Guéno et Laplume (57).

${ }^{3}$ Henri Barbusse, cité par Rousseau (122).

${ }^{4}$ Paul Wattelet (pseudonyme de Louis Aragon) in Confluences, 21-24 (1943) : 414-415 ; cité par Rieuneau (165).

${ }^{5}$ Martin Vaillagou, cité par Guéno et Laplume (46).

${ }^{6}$ Rieuneau (13).

${ }^{7}$ Benjamin Crémieux, cité par Rousseau (186).

${ }^{8}$ Rieuneau (20).

${ }^{9}$ Cendrars (317).

${ }^{10}$ Gaston Biron, cité par Guéno et Laplume (104)

${ }^{11}$ Julien Benda, «La Crise de la vérité », Les Nouvelles littéraires. 8 novembre 1930 ; cité par Rousseau (226) 